\title{
A User Study on Kinesthetic Teaching of Redundant Robots in Task and Configuration Space
}

\author{
Sebastian Wrede, Christian Emmerich, Ricarda Grünberg, Arne \\ Nordmann, Agnes Swadzba, and Jochen Steil \\ CoR-Lab, Bielefeld University
}

The recent advent of compliant and kinematically redundant robots poses new research challenges for human-robot interaction. While these robots provide a great degree of flexibility for the realization of complex applications, the flexibility gained generates the need for additional modeling steps and definition of criteria for redundancy resolution constraining the robot's movement generation. The explicit modeling of such criteria usually require experts to adapt the robot's movement generation subsystem. A typical way of dealing with this configuration challenge is to utilize kinesthetic teaching by guiding the robot to implicitly model the specific constraints in task and configuration space. We argue that current programming-by-demonstration approaches are not efficient for kinesthetic teaching of redundant robots and show that typical teach-in procedures are too complex for novice users. In order to enable non-experts to master the configuration and programming of a redundant robot in the presence of non-trivial constraints such as confined spaces, we propose a new interaction scheme combining kinesthetic teaching and learning within an integrated system architecture. We evaluated this approach in a user study with 49 industrial workers at HARTING, a medium-sized manufacturing company. The results show that the interaction concepts implemented on a KUKA Lightweight Robot IV are easy to handle for novice users, demonstrate the feasibility of kinesthetic teaching for implicit constraint modeling in configuration space, and yield significantly improved performance for the teach-in of trajectories in task space.

Keywords: Physical human-robot interaction, compliant robots, redundant robots, kinesthetic teaching, impedance control, learning, assistance systems, field study.

\section{Introduction}

A new generation of adept manipulators has recently become more widely used, combining two technological advancements: advanced force-torque sensing integrated into compact actuation units (Albu-Schäffer, Haddadin, et al., 2007) with variable stiffness (Tsagarakis, Sardellitti, \& Caldwell, 2011) leading to fully compliant robot manipulators (Bischoff et al., 2010; Grzesiak, Becker, \& Verl, 2011) and the tendency to increase the degrees of freedom towards kinematically redundant (Conkur \& Buckingham, 1997) manipulators. While these robots provide a great degree of flexibility for the realization of complex applications - for example, in industrial (Daimler AG,

\footnotetext{
Authors retain copyright and grant the Journal of Human-Robot Interaction right of first publication with the work simultaneously licensed under a Creative Commons Attribution License that allows others to share the work with an acknowledgement of the work's authorship and initial publication in this journal.
}

Journal of Human-Robot Interaction, Vol. 2, No. 1, 2013, Pages 56-81. DOI 10.5898/JHRI.2.1.Wrede 
2009) or service robotics (Akgun, Cakmak, Yoo, \& Thomaz, 2012; Breazeal et al., 2008) scenarios - the gained flexibility generates the need for additional modeling steps and the definition of criteria for redundancy resolution.

The required models for these specific criteria (e.g., to exploit the dexterity of a redundant robot to fulfill a task within complex environmental constraints) are not easily accessible to end users and requires experts to configure and - in the case of a change of tasks - to reprogram the robot. The potential benefits of using redundant robots are thus often reduced through high costs for the tedious adaptation of the underlying control processes. It has often been proposed that learning by demonstration is a solution to this problem (Schaal, Ijspeert, \& Billard, 2003), and kinesthetic teaching (i.e., physical guidance of the robot by the users) has been considered as an interface for providing demonstrations (Billard, Calinon, Dillmann, \& Schaal, 2007). In this context, kinesthetic teaching typically provides data for learning task-space movements (Calinon \& Billard, 2007a) or a particular task trajectory, including a redundancy resolution (Vijayakumar, D'souza, Shibata, Conradt, \& Schaal, 2002).

These approaches to kinesthetic teaching do not reflect the standard technical approaches to redundancy control, which typically separate task-space planning and constraint resolution in configuration space. Furthermore, dedicated interaction support for kinesthetic teaching of constraints in confined spaces has not yet been considered. That is, standard robot control distinguishes task and configuration space in the redundancy resolution, but users have to deal with the complexity of simultaneously considering the redundancy resolution in configuration space and the specification of trajectories in task space. Therefore, our hypothesis is that kinesthetic teaching in confined spaces, with the goal of avoiding explicit modeling of constraints in the configuration space in addition to the task, is indeed challenging for non-experts if redundancy resolution is not trivial. Based on these considerations, a primary goal of this contribution was to evaluate in a user study whether kinesthetic teaching of constraints in configuration space is possible and if a separation of task- and configuration-space interaction provides benefits compared to the typical teach-in methods that do not consider this separation. These ideas, and the attractive interaction properties of compliant and redundant robots for kinesthetic teaching, motivated us to develop an interactive robotics system explicitly distinguishing between configuration- and task-space interaction.

A secondary goal of the study was to assess if this system concept reduces the complexity of adapting a redundant robot to new environments and tasks. It combines physical human-robot interaction (pHRI) (De Santis, Siciliano, De Luca, \& Bicchi, 2008; Alami et al., 2006) in terms of kinesthetic teaching and learning methods in an integrated approach. It aims at enabling users with no particular robotics knowledge to perform the required steps for environment-specific configuration and task-dependent programming of redundant robots within a few minutes. The logical distinction between configuration- and task-space interaction allows the decomposition of the overall adaptation process into two separate but consecutive phases: The first phase is the configuration phase, where kinesthetic teaching is used to implicitly model obstacles or task-independent constraints, which are relevant for movement generation in the joint space of the robot. Here, constraint knowledge is implicitly transferred through application of a learning method from the human tutor to the robot system without explicit constraint modeling. The learned inverse kinematics mapping is embedded in a hierarchical controller (Nordmann, Emmerich, et al., 2012), allowing for execution of arbitrary motions in the task-space but respecting the learned constraints. The second phase is the programming phase where the human tutor kinesthetically teaches a specific task-space trajectory to the robot just by applying forces to the end-effector. The robot's joints are controlled in this phase by the hierarchical controller trained in the previous phase. This allows the users to program a specific task without consideration of the environment. We refer to this control mode as Assisted Gravity Compensation. 


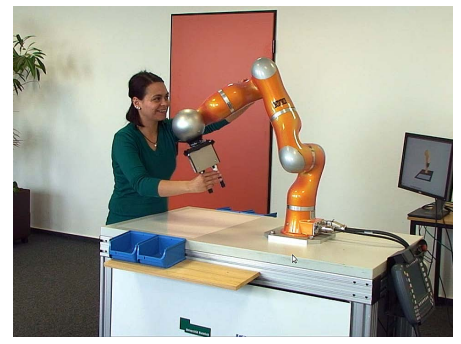

(a) Warm-up interaction

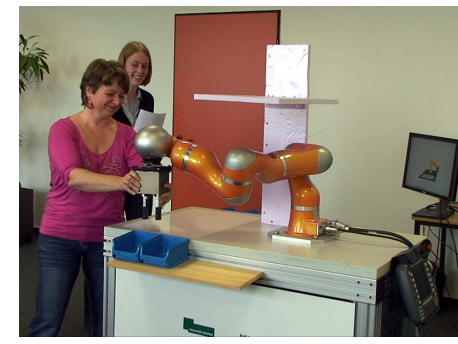

(b) Configuration phase

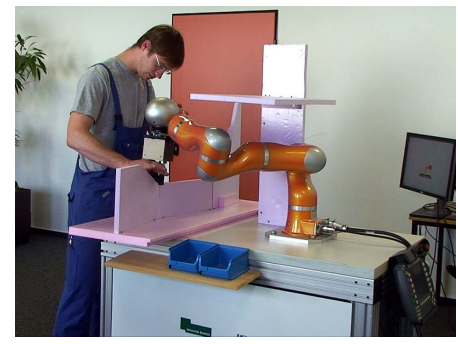

(c) Programming phase

Figure 1. Typical interaction with the FlexIRob system during the experimental study at HARTING.

The main benefits expected from the proposed two-step process are that (i) task-independent constraints for the redundancy resolution can be configured by laypersons through kinesthetic teaching such that, for instance, obstacles in the joint-space are avoided without explicit environment modeling; (ii) the programming of task-space trajectories can be done faster and more accurately than with existing procedures; and (iii) the cognitive load and physical effort are reduced through splitting the overall task into the two consecutive interaction phases.

The interaction approach and its anticipated benefits were evaluated in a user study with 49 participants at a medium-sized manufacturing company - the HARTING technology group ${ }^{1}$ - in Germany. Most of the participants had no practical experience with robots. Within the study each of the participants had to configure constraints for a selected environment - after a short warm-up phase - and program a reference task (cf. Figure 1). Besides the evaluation of the general interaction experience with the compliant robot system, both the configuration and the programming phases were evaluated in detail. For the latter, we split the participants into two groups. One group had to teach the robot a task in the assisted gravity compensation mode, while the control group used a typical teach-in procedure - as provided by the robot manufacturer - requiring the simultaneous consideration of the task specifics and the environmental constraints reflected in certain joint-space configurations. To the best of our knowledge no such study on kinesthetic teaching for reconfiguration of redundant robots has been carried out with laypersons so far.

The article is structured as follows: Related work on interactive programming of redundant robots, kinesthetic teaching and respective system studies is discussed in Section 2. Section 3 explains the experimental robot system implementing the described interaction concepts, termed FlexIRob, from a technical perspective. Section 4 explains the design of the systems study we conducted, and Section 5 reports the results gathered through a questionnaire and quantitative analysis of system-level performance data. Section 6 discusses these results, while Section 7 presents a brief summary and an outlook on future research challenges.

\section{Related Work}

Facilitating efficient reconfiguration of non-trivial robot systems is an acknowledged challenge (European Strategic Robotics Platform, 2009) of current robotics research. In the following, related work is presented corresponding to the configuration phase, which mainly considers the modeling of constraints at the kinematics level, and prior work addressing the programming phase that is concerned with teaching trajectories at the task level as well as combined approaches.

\footnotetext{
${ }^{1} \mathrm{http}: / /$ www.harting.com
} 
The configuration of redundant robots in terms of constraining their movement generation at the kinematics level is typically carried out as an offline process without any direct human-robot interaction. Offline methods specifically enforce constraints defined in advance of system runtime and require explicit modeling; for example, by directly modifying the implementation of the inverse kinematics solution or through optimizing the parameters of an iterative redundancy resolution process. Typical approaches include the selection of single pre-defined redundancy solutions, the minimization of an energy function (Siciliano, 1990), or are based on linear programming methods (Lalo, Brandt, Schramm, \& Hiller, 2008). In the latter approaches, null-space configurations are selected based on additional criteria such as "home" positions, joint angle, and velocity or acceleration limits, which are examples for constraints that need to be explicitly modeled upon every reconfiguration of a redundant robot system. Alternative schemes realize biomimetic models, such as the Bayesian modeling techniques used by Artemiadis, Katsiaris, and Kyriakopoulos (2010) to build an inverse kinematics solver preferring human-like joint configurations for redundancy resolution of humanoid robot arms.

In contrast to analytic approaches, an important area of work is concerned with learning inverse models for redundant robots. However, only a limited number of these models consider the interactive introduction of additional constraints for redundancy resolution. An exemplary approach in this category is constrained supervised learning (Jordan, 1992) which explicitly models configuration and temporal constraints for solving inverse kinematics problems. While this is conceptually similar to the learning process presently integrated into the FlexIRob system, the actual constraints are not learned from training data but have to be explicitly specified. More recently, Neumann, Rolf, and Steil (2010) utilized a learning scheme on a humanoid robot similar to that used in this contribution for pose-constraint bi-manual movements. Despite the similarity in the learning method, in that case the pose constraint was explicitly modeled beforehand, and no kinesthetic teaching of the constraint was involved.

While learning in interaction is rather rare at the kinematics level and many methods require offline configuration, online methods for programming actual tasks at the trajectory level realize some kind of teach-in procedure; for example, through tele-operated (Levas \& Selfridge, 1984) interaction or direct pHRI, typically by mounting a force/torque sensor at specific joints or the end-effector of a robot (Infante \& Kyrki, 2011). Other approaches monitor with external sensing the actions of a human tutor and apply imitation learning methods to teach more complex tasks interactively (Billard et al., 2007; Kulic, Ott, Lee, Ishikawa, \& Nakamura, 2011; Muehlig, Steil, \& Gienger, 2012; Schaal et al., 2003) and, partially, also incrementally (Calinon \& Billard, 2007b) in order to derive criteria to select a particular redundancy resolution.

A recent example for a motion generation approach based on hidden Markov models integrating kinesthetic teaching is presented in Lee and Ott (2010). An advanced interaction concept allows iterative refinement of motion primitives subsequent to observational learning of a reference trajectory. Another example of motion learning and adaptation through kinesthetic teaching combines imitation learning and an adaptive control algorithm (Gribovskaya, Kheddar, \& Billard, 2011), facilitating adaptive impedance control of robot motions in human-robot collaboration scenarios. Ito and Tani (2004) present a related approach based on online imitation learning inspired by the idea of mirror neurons. Through observation of a human tutor movement trajectories are learned and encoded in a recurrent neural network model. A particular benefit of their method is that generation and recognition of motion patterns is supported. While these approaches allow sophisticated programming of task-specific robot movements, redundancy resolution specified implicitly for a given environment or class of tasks in one demonstration has to be taught repetitively in all following demonstrations and can not be generalized. 
The aforementioned methods either focus on constraint modeling for redundancy resolution and inverse kinematics mapping or consider task-space trajectories. Combined approaches allow configuration of constraints for movement generation and program task trajectories simultaneously through kinesthetic teaching. For instance, the commercially available KUKA Lightweight Robot (Bischoff et al., 2010) has features that allow the recording of artificial joint trajectories through kinesthetic teaching in a gravitation compensation mode or through a series of postures set interactively (through tele-operation or in physical interaction), which are used as key frames for the generated robot movement. A similar approach was conducted on the service robot Simon to teach new skills and tasks in interaction (Akgun et al., 2012). Both approaches allow one to make use of the full redundancy of the robot. That said, our assumption is that this concept has several drawbacks; for example, high cognitive load for the human tutors as they need to consider the required redundancy resolution and the task specification simultaneously throughout the reconfiguration process. Furthermore, the implicit encoding of the redundancy resolution has to be taught repetitively in every demonstration, as in the aforementioned task-level imitation learning approaches. A more sophisticated example for a combined approach following a programming-by-demonstration scheme for configuration and programming is to exploit imitation learning techniques for teaching task trajectories and subsequently use tactile feedback to refine the movement generation according to further constraints (Sauser, Argall, Metta, \& Billard, 2012). While this approach is applicable in free space, it will be impossible to apply in confined spaces where the initial redundancy resolution must already comply with the constraints of the environment.

The approaches presented above consider either the configuration or the programming phases individually, while only a few approaches report on the combination of both. Configuration is usually carried out by explicit constraint modeling or through machine learning, without consideration of human-robot interaction. Programming of redundant robots through kinesthetic teaching in terms of imitation learning or direct replay seems intuitive and appealing at first sight. The work reported in this contribution shows that improvements to the underlying human-robot interaction scheme are required to increase the usability, efficiency and accuracy of the programming procedure. We present a new interaction concept, which is evaluated in a systems study simplifying both the configuration and programming of redundant robots in a combined approach.

\section{The FlexIRob System}

Motivated by the identified challenges in the reconfiguration of redundant robots and as the main experimental tool in our user study, we facilitate our interactive robot system, termed FlexIRob, which will be briefly introduced in this section. Its implementation realizes the separate but consecutive interaction phases sketched in the introduction but also gives easy access to control modes typically used for kinesthetic teaching. The remainder of this section introduces the compliant robot platform, the interaction control modes, and their associated control flow, as well as the work flow designed to easily allow interactive configuration and to assist users during programming.

\subsection{Compliant Robot Platform}

We developed our interaction system using a recent version of the KUKA Lightweight Robot (LWR IV) (Bischoff et al., 2010), which is a redundant manipulator with seven joints, allowing a manifold of configurations in joint space for a single end-effector position. We use the LWR IV as a basis for the FlexIRob system due to its impedance-based control scheme (Albu-Schäffer, Ott, \& Hirzinger, 2007), resulting in active compliance of the manipulator. Other control modes, although available on this platform, are not used in this study to avoid interrupting the interaction for control mode switches. While physical interaction is also possible with conventional industrial robots with six degrees of freedom if force sensors are added at the end-effector, only the redundancy of the 


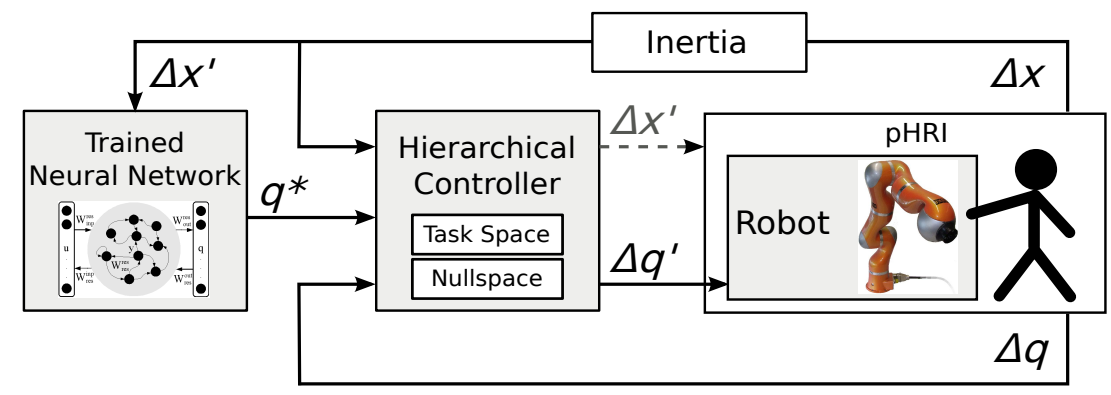

Figure 2. Block diagram showing the FlexIRob control scheme during the assisted gravity compensation mode. The resulting joint command $\Delta q^{\prime}$ corresponds to the end-effector deviation $\Delta x^{\prime}$, which is based on the physical Human-Robot Interaction, but with respect to the redundancy resolution encoded in the neural network.

LWR IV in combination with the force sensors in each of the joints allows intuitive teaching along the entire joint configuration via physical interaction.

\subsection{Interaction Controller}

For the different interaction steps of the configuration and programming phases, we defined a set of specific interaction control modes. All of these modes are based on the joint impedance mode of the LWR IV, which provides the necessary flexibility for all of our desired interaction modes by using different, task-dependent stiffness and damping values (see Table 5). Five interaction control modes were used in this study:

3.2.1 Compliant execution executes executes position commands and trajectories in joint space. Impedance values are chosen to make precise movements, but also allow physical interaction (e.g. online trajectory corrections) during execution.

3.2.2 Controlled gravity compensation mimics the built-in gravity compensation mode of the LWR IV. The robot is compliant so that the user can move the robot through physical interaction and the reference for the joint impedance controller continuously tracks the current position of the robot. Hence the robot is freely movable in all its axes, but hold its position if no external force is applied. The ease of moving is determined by the stiffness and damping values of the underlying joint impedance control of the LWR IV. An additional inertia term in Cartesian space prevents the robot from continued drifting after being moved.

3.2.3 Compliant recording holds the robot in its current position to allow recording of training data in the vicinity of a fixed-joint configuration. The underlying joint impedance allows the user to move the robot while it remains in its basic configuration. End-effector positions and the associated joint angles are recorded for supervised training of a neural network. The stiffness and damping values were carefully chosen to allow the exploration of a reasonable vicinity around the fixed configuration without being too sensitive, potentially leading to noisy and inconsistent training data.

3.2.4 Assisted gravity compensation assists users during the programming phase and is compared to the basic controlled gravity compensation mode in the system study. Figure 2 shows the control flow of the assisted gravity compensation mode. As described in Section 3.2.2, applying an interaction force to the robot's end-effector results in changing the Cartesian end-effector position by $\Delta x$, 


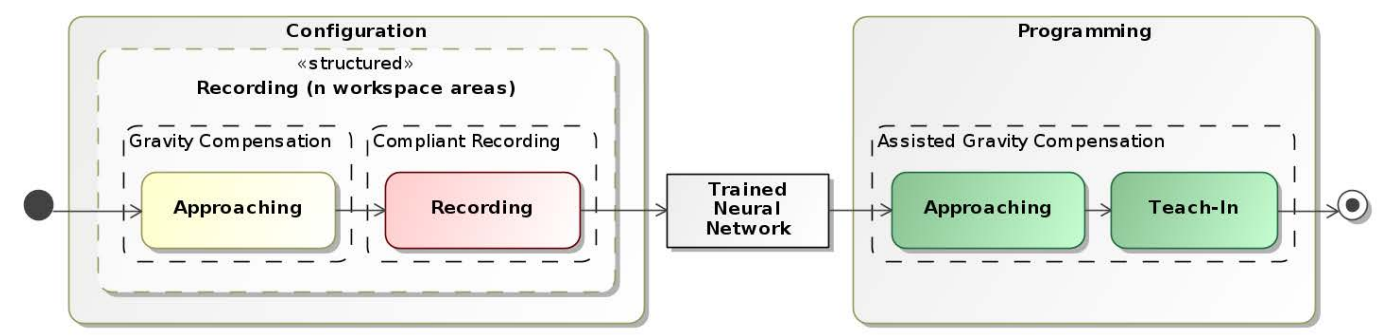

Figure 3. Interaction work-flow with assisted teach-in using the interaction control modes: controlled gravity compensation (yellow box), compliant recording (red box) and assisted gravity compensation (green boxes). Recording of the training data can be repeated for several workspace areas. The trained neural network from the configuration phase is transferred to the programming phase to be used inside the assisted gravity compensation mode.

which is passed to an inertia term to generate the Cartesian reference $\Delta x^{\prime}$. To simultaneously assist the user during programming, assisted gravity compensation additionally controls the joint configuration by using a given redundancy resolution encoded in a neural network. The neural network generates joint position references $q^{*}$ for $\Delta x^{\prime}$ according to its encoded redundancy resolution. In order to fuse tracking in task space $\Delta x^{\prime}$ and joint-space control of $q^{*}$, we use a hierarchical position controller, based on ideas by (Grupen \& Huber, 2005). The hierarchical controller prioritizes the end-effector task $\Delta x^{\prime}$, while complying as far as possible with the joint-space task $q^{*}$, given by the redundancy resolution. To assist the user in this mode, the robot is freely movable in task space, but controlled in joint space according to the desired redundancy resolution to assist the user.

3.2.5 Hierarchical control (Nordmann, Emmerich, et al., 2012) controls the Cartesian endeffector position, as well as the joint configuration according to a given redundancy resolution. Similar to the assisted gravity compensation, it uses the redundancy resolution encoded in the trained neural network mode to generate joint position reference $q^{*}$ for a Cartesian task $x$. However, $x$ is not given by physical Human-Robot Interaction in this mode but can be an arbitrary Cartesian position command or trajectory. As in the assisted gravity compensation mode, $q^{*}$ and $x$ are fused by a hierarchical controller. Hence, the robot executes arbitrary movements in the task space respecting the desired redundancy resolution, which is encoded in the neural network.

\subsection{Interaction Workflow}

The interaction work flow, separated into configuration by implicit modeling of redundancy resolution on the one hand and assisted programming on the other hand, is depicted in Figure 3. The first step in the configuration phase is approaching the work space area where a desired redundancy resolution will be taught. The robot is set to the controlled gravity compensation mode to be freely movable in its entire joint configuration. Once the desired joint configuration for this workspace area is applied by the user, the robot is switched to the compliant recording mode. The user can now start recording training data by applying force to the end-effector.

These two steps can be repeated for an arbitrary number of different workspace areas to kinesthetically teach different redundancy resolutions for different areas of the robot's workspace. The recorded joint angles $q$ and corresponding Cartesian end-effector positions $x$ are used for supervised training of the neural network. (In principle, any other trainable function approximator with reasonable inter- and extrapolation capabilities can be used.) The trained neural network then encodes an inverse kinematics with the desired redundancy resolution for all workspace areas and is sub- 
sequently used in the programming phase, to be included in the assisted gravity compensation and hierarchical control modes.

The programming phase starts again with approaching the teach-in task, this time in the assisted gravity compensation mode. While approaching and during the subsequent teach-in, the user no longer needs to care for the redundancy resolution, which is now done by the control mode according to the constraints that were implicitly encoded in the neural network during the configuration phase.

\subsection{Software Architecture and Safety}

The interaction system presented in this chapter is implemented as a coherent set of learning, interaction, and control components with the Compliant Control Architecture (Nordmann, Rolf, \& Wrede, 2012). Lower-level control components run inside a soft real-time execution engine implemented as a Xenomai task in user space, which is interfaced with KUKA's Fast Research Interface (FRI) (Schreiber, Stemmer, \& Bischoff, 2010). Due to the risks in physical contact between the robot and human users, we applied a number of safety measures to ensure safety for humans on the one hand and allow research in system studies on the other hand.

The main risk is collision between a robot and its user (Alami et al., 2006). The first means to reduce the instantaneous severity of impacts is the use of the KUKA Lightweight Robot IV, which reduces manipulator link inertia and weight by using lightweight but stiff materials. The interaction control modes introduced above and used throughout the experiments are compliant to external forces which also reduces impact in case of a collision. Low-level control components and the FRI-based communication checks monitor the quality of service of the network communication, providing reliable robot control at all time. The control system immediately stops the robot when communication quality drops. Limits for joint positions, velocities, and acceleration were configured and are constantly checked on different layers of the software architecture to prevent injuries from collisions or shearing. The hierarchical controller used in the hierarchical control and assisted gravity compensation modes deals with the uncertainty of insufficient or inadequate training of the system by prioritizing control of the end-effector trajectory and therefore preventing unexpected end-effector movements of the robot.

\section{Study Design}

Based on our assumption that it is difficult for a human tutor to simultaneously take care of the end-effector position and all joints of the manipulator in confined spaces as well as the available interaction modes realized in the FlexIRob system, we designed a user study to validate our hypotheses and gain further insights. The study was designed along the proposed decomposition of the overall reconfiguration interaction into a task-independent configuration and a task-dependent programming phase. The high-level objective of the study was to evaluate this interaction scheme with industrial workers and validate if kinesthetic teaching and learning methods are usable in the chosen design for inexperienced users. Participants of the study were recruited from HARTING, a company endorsing its assembly line for automatic production with manual assembly for small lot sizes. The company planned for 50 workers to take part in the study, with 49 eventually participating.

The large number of study participants allowed us to examine in detail the implications of the proposed concept in terms of the following three questions:

(i) What is the general experience with our FlexIRob system?

We expect that the physical interaction is:

- easy and comfortable

- not threatening, and 
- self-explanatory especially for people with good spatial vision abilities.

(ii) How feasible is the task-independent configuration for naive users?

We hypothesize that it is possible to transfer one's own knowledge of the environment to the robot via kinesthetic teaching. This hypothesis will be analyzed along the following criteria:

- How fast can the configuration be done (e.g., in less than 5 min.)?

- Are collisions avoided?

- How accurate is the configuration?

(iii) How helpful is the decomposition of the interaction into a task-independent configuration and a task-dependent programming phase by means of the new assisted gravity compensation mode?

As this is our central research question, it is reflected in the conditions of our study. In the one condition, the participant performs a teach-in task supported by the robot in respecting the constraints of the environment. In the other condition, the task needs to be solved without assistance. We hypothesize that

- the assisted group has a better interaction experience than the non-assisted group and

- the assisted mode allows a faster, more precise, and collision-free teach-in.

\subsection{Study Setup}

According to the proposed decomposition of robot teaching into a task independent configuration and a task-dependent programming, our study consists of two main parts, depicted in Figure 4. The first part focuses on teaching the robot desired redundancy resolutions respecting the constraints from the environment in relevant regions of the task space. We restrict the setting to two regions as illustrated in Figure 5, in order to keep complexity low, although from the learning point of view any number of regions would be possible. In our study the constrained environment was established by a Styrofoam construction as shown in Figure 1(b). The second part deals with a teach-in of a concrete task in this environment. As industrial tasks often require that a robot arm be able to follow a demonstrated trajectory, we had them play a variant of the wire-loop game ${ }^{2}$ to simulate this ability. Participants had to teach the robot a trajectory along the Styrofoam parcours placed in the constrained working space (see Figure 1(c)). To test the hypothesis that the assisted gravity compensation mode reduces the task complexity, we divided our study participants into two groups. Group A was supported by the assisted gravity compensation mode; participants only needed to guide the end-effector. Group $\mathrm{N}$ was not actively assisted by the robot and used the Controlled Gravity Compensation mode. Participants needed to take care of all joints and the end-effector simultaneously in order to avoid collisions with the environment while also performing the task programming. The experiment was thus divided into four parts:

1. a warm-up phase,

2. the task-independent configuration phase (3 trials; see Figure 5)

3. the wire-loop game (see Figure 1(c))

4. the questionnaire

The interaction work-flow of phases $1-3$ is illustrated in Figure 4 and depictions of these phases are provided in Figure 1.

\footnotetext{
${ }^{2}$ The original wire-loop game consists of a metal loop and a serpentine length of wire connected to a power source. The loop has to be guided along the wire without touching it. If loop and wire touch a closed electric circuit is formed and a lightor sound-emitting device indicates that the game is lost.
} 


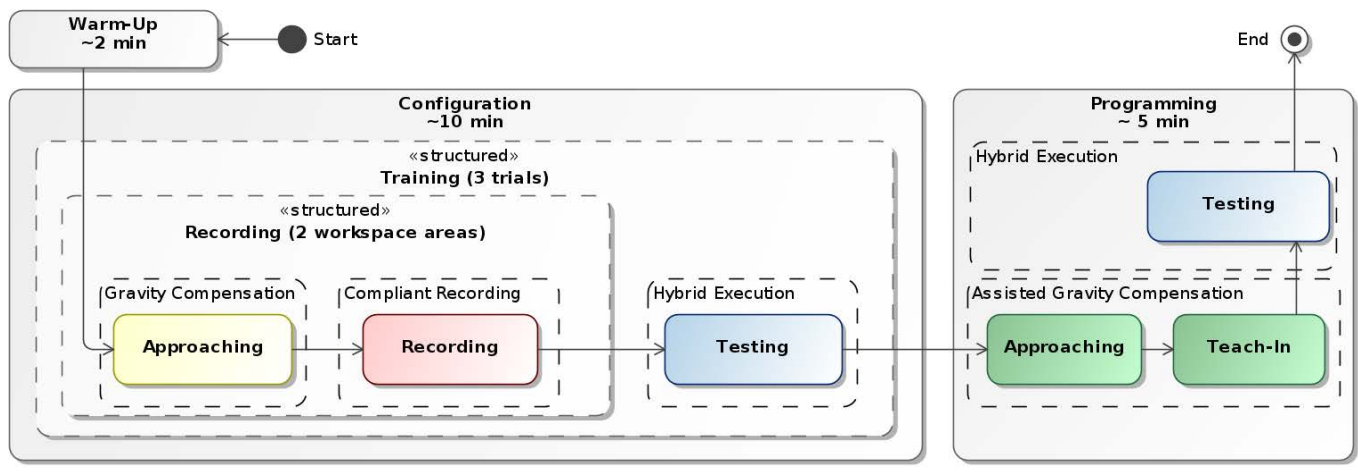

Figure 4. Interaction work-flow during the study of the assisted group, using the introduced control modes controlled gravity compensation (yellow box), compliant recording (red box), hierarchical control (blue boxes) and assisted gravity compensation (green boxes). The Configuration phase consists of three trials, each of which includes training the robot in two workspace areas. After each trial, the robot is executed in hierarchical control with the trained network to provide feedback. The Programming phase is done once in assisted gravity compensation (controlled gravity compensation for the non-assisted group), and also ended with feedback through execution in hierarchical control.

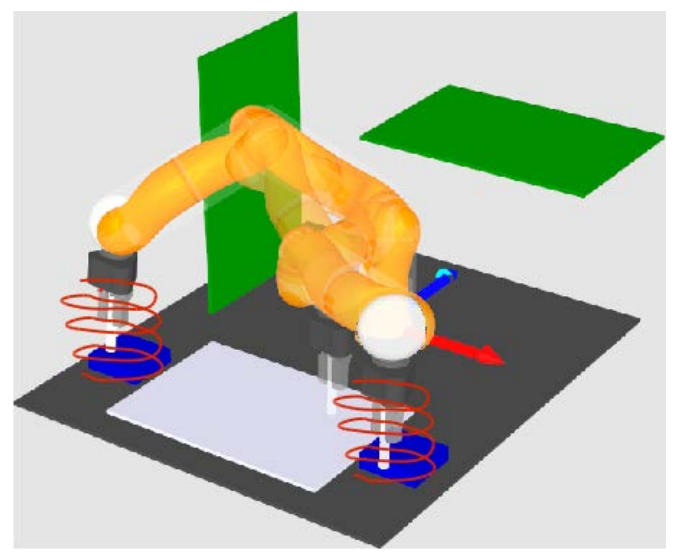

Figure 5. Schematic illustration of the steps carried out during the configuration phase, which are as follows: training the left working area by performing circular movements, moving the arm to the right working area, and training the right working area by moving the robot's end-effector again in a helix-like fashion. During training the arm is controlled by the compliant recording and during transition by the controlled gravity compensation mode. The rectangles to the left and above the robot provide environmental constraints for testing the system in simulation.

4.1.1 Warm-up phase The experiment started with welcoming of the participants and an overview of the procedure. They were asked to sign an informed consent form and a consent form for recording and later usage of the video data. This was completely voluntary and could be declined. After that the warm-up was started. First, participants watched an instructional video ${ }^{3}$ showing how all seven joints could be moved and how the arm could be moved into different positions. They were informed about the two main operational modes of the robot system, the controlled gravity compensation and the compliant recording (see Section 3.2). To switch between the two modes the participants just needed to leave the arm for a short amount of time, which automatically switches the system to the other mode after a short motion pause is detected. The mode switching is indicated by a short audio prompt. The participants were also told that they could not damage anything and could safely touch the robots' gripper. Afterwards, the participants were instructed to familiarize themselves with the robot. More specifically, they were asked to reproduce what they saw in the

${ }^{3}$ http://www.cor-lab.de/system/files/Instruktion.mp4 
video. Depending on what participants tried on their own, they were encouraged to try both operational modes and different movements, to make sure that they were prepared for the following tasks.

4.1.2 Configuration phase After participants had finished the warm-up, they were asked to perform the first task. During the configuration phase a model work place with a confined working space for the robot was set up (see Figure 1(b)), and the participants were asked to kinesthetically teach a redundancy resolution to the FlexIRob system within this confined working space. First, they had to move the robot to the left working area and were instructed to perform circular movements for training this area. Then, they had to move the arm to the right working area where the teaching procedure was repeated (cf. Figure 5 for an illustration of the reconfiguration phase). To investigate how much instruction participants needed to complete the task successfully (in the sense of providing reasonable training data in both regions of the workspace), configuration was repeated in three trials with increasingly informative instructions, which were the same for all participants:

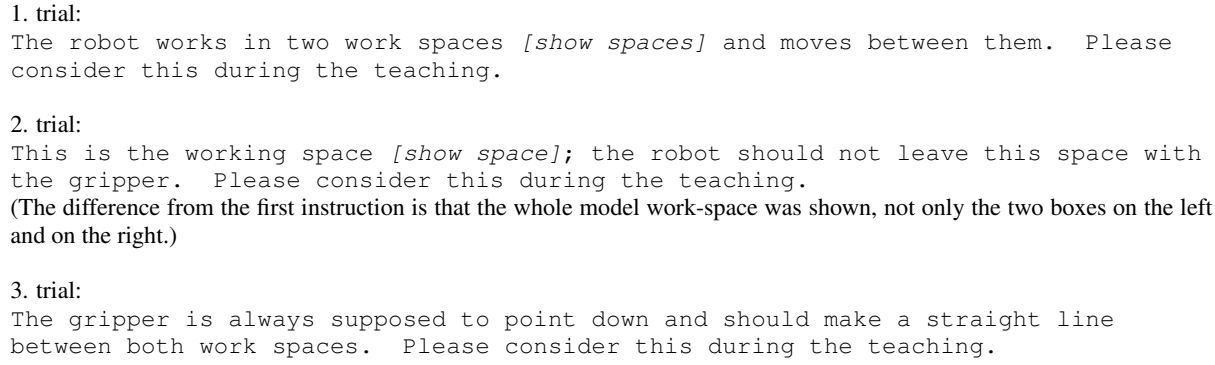

The hints were chosen in such a way that with ongoing trials the possibilities for moving the robot from one working area to the other were more and more restricted, and thus should help the participants to find a redundancy resolution where the arm can move in a straight line from the left to the right working area.

After each trial the, participant was told to step back from the robot. Once the system was trained, a reference movement (a straight line predefined in the task space for this experiment) was performed by the robot arm from the left to the right training area to give basic feedback to the participant about the resulting validity of the learned control scheme. The task was then repeated and the next instruction was given.

4.1.3 Wire-loop game The last part of the study was the wire-loop game simulating a teach-in of a concrete task to the robot. During this task the experimental manipulation took place. Participants were randomly assigned to group $\mathrm{N}$, where they had to program the robot in the controlled gravity compensation mode without any assistance by the system, or to group A, which was supported during task programming through the assisted gravity compensation mode. In order to get reproducible results, all assisted participants started with a reference neural network trained by an expert in advance. After the teach-in, participants were told to step back and watch how well the teach-in worked. The robot then exactly copied the way the participants had guided it.

4.1.4 Subjective assessment of interaction Finally, the participants were given the questionnaire (cf. Section 4.2). They were instructed how to fill in the questionnaire and were given a short overview of the different topics. They were instructed to ask if there were any questions they did not understand. After the completion of the questionnaire participants were debriefed. To keep the conditions and instructions stable through the whole experiment each conductor of the study adhered to a set of guidelines ${ }^{4}$ during the experiment.

\footnotetext{
${ }^{4}$ Full instructions available at: http://www.cor-lab.de/system/files/instructions.pdf
} 


\subsection{Questionnaire Design}

The questionnaire was adapted to the tasks and to the specific demographic background of the participants. Characteristics of the sample included educational background and the fact that some employees were not native German speakers. The questions were derived from expert-discussions and pre-tested with four students from Bielefeld University. Due to the characteristics of the sample, all questions were also discussed with a member of the staff of HARTING. The resulting questionnaire contained 36 questions structured into seven topics:

- general experience with the robot during the interaction

- subjective experience of the wire-loop game

- whether or not participants could imagine the robot supporting them during various tasks

- demographic variables

- other control variables (e.g. stereoscopic vision)

- previous experience with robots

- suggestions/ ideas for improvement

The items concerning general experience covered important characteristics of the robot, like threat, reliability, and intelligence; and characteristics of the handling, like ease, pleasantness, cognitive load during handling, etc. All items concerning the robot and the task were rated on a five-point Likert scale ranging from 1 (yes/very much) to 5 (no/not at all). All items covering previous experience, support by the robot and control variables were rated on a three-point Likert scale ranging from 1 (yes/very much) to 3 (no/not at all). This reduction of dimensions was used to facilitate the completion of the questionnaire 5 .

\section{Experimental Results}

This section is organized along three dimensions. First, results from the analysis of the questionnaire are presented in Section 5.1. Second, we take a deeper look into the configuration phase in Section 5.2. And last, in Section 5.3 the effect of the assisted gravity compensation mode is analyzed in the context of the wire-loop game. Inputs to the analysis included both subjective assessments by the participants gathered through the questionnaire and objective measurements, as well as derived metrics based on the system data recorded during the interaction experiments with our data logging toolchain (Wienke, Klotz, \& Wrede, 2012).

\subsection{Questionnaire Analysis}

In the following, the results from the analysis of the questionnaire are presented. We start with a brief summary of the participants' demographics and a presentation of descriptive results about their user experience. Subsequently, we present the results of the participants' experiences during the programming phase (wire-loop game) concerning the two conditions (assisted and non-assisted). Finally, we report some interesting results concerning other variables that occurred to influence the subjects' experience.

5.1.1 The participants The study was planned for 50 participants; 49 of them participated and one was ill. The final sample consists of 44 participants, 15 males and 29 females. Participants were excluded when failures occurred that demanded a system restart. Figure 6 lists the participants' working units, their native language, and their educational background. The participants' ages ranged from 25 to 55 , with an average of 41.34 years $(\mathrm{SD}=8.73)$. The working time at HARTING ranged from 1 to 33 years, with an average of 10.86 years ( $\mathrm{SD}=9.68)$. Concerning spatial vision $72.2 \%$ reported having good spatial vision, $25 \%$ reported moderate spatial vision and

${ }^{5}$ Available at: http://www.cor-lab.de/system/files/FragebogenHartingstudy.pdf 
$2.3 \%$ reported bad spatial vision. With regard to their familiarity with robots, $79.5 \%$ of participants reported knowing robots from TV, newspapers, or other sources, while $20.5 \%$ reported not knowing any robots; $59.1 \%$ reported knowing industrial robots and $40.1 \%$ did not know industrial robots. As to experiences with robots only, $2.3 \%$ of participants had prior experience with robots, $13.6 \%$ reported a bit of experience with robots, and the majority, $84.1 \%$ of participants, had no prior experience with robots at all. Participants were randomly assigned to the two conditions. The final data set consisted of 21 participants in the non-assisted condition and 23 in the assisted condition.

5.1.2 Descriptive results The results for the general experience with the robot during the experiment are shown in Table 1. A rating of 1 indicates yes/very much and 5 indicates no/not at all. A very important result is that participants rated the robot as non-threatening, although, depending on which department of HARTING they work in, they get safety instructions and are warned against the dangers of industrial robots. One reason for participants' experience of the robot as non-threatening might be that they also rated the system as reliable. Another very distinct and important rating is that the participants perceived the robot's intelligence to be high, and they also felt the acoustic and visual feedback given by our system was helpful to complete the tasks. In addition, the overall handling of the robot was rated to be easy.

Table 1 also shows the results concerning the participants' ideas about how this robot system could assist them during their own work. The results indicate that participants thought assistance in holding heavy parts and putting parts away is most realistic, while collaboratively screwing parts in was seen as less realistic. This might be due to the fact that the gripper used in the study was not seen as suitable for tasks that require fine motor skills.

5.1.3 Main effects regarding the condition As already mentioned, participants were divided into two groups: One group performed the task (wire-loop game) in gravity compensation mode and was not assisted by the system, and the other group performed the task in assisted gravity compensation mode, during which the joint configuration was controlled by the system.

Programming Phase. We assumed that the group that was assisted by the system would find the task programming easier and more pleasant and would rate the operation mode more reasonable than the group without assistance from the system. To test these hypotheses, an analysis of variance (ANOVA) was computed with condition as the independent variable and the variables concerning the simplicity and pleasantness of the task and the reasonableness of the control mode as dependent variables. For the sake of readability, only means and standard deviations are described in the text; for other relevant values see Table 2 . The ANOVA revealed a highly significant effect on the simplicity of handling. Participants in the non-assisted mode found the handling significantly less easy $(\mathrm{M}=2.52, \mathrm{SD}=1.12)$ than did participants in the assisted mode $(\mathrm{M}=1.70, \mathrm{SD}=0.71)$.
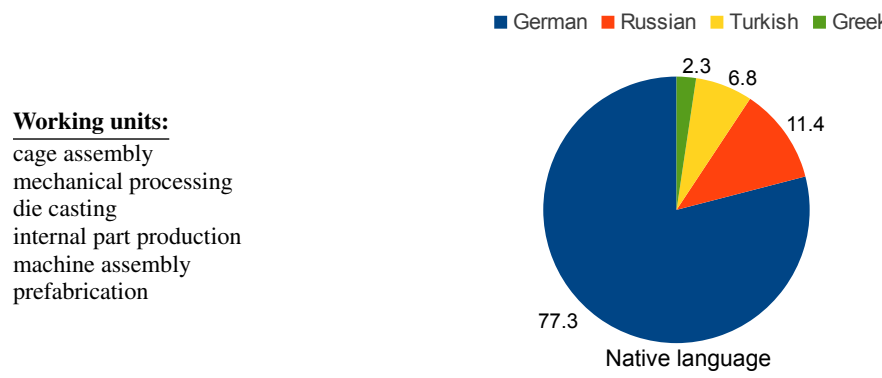

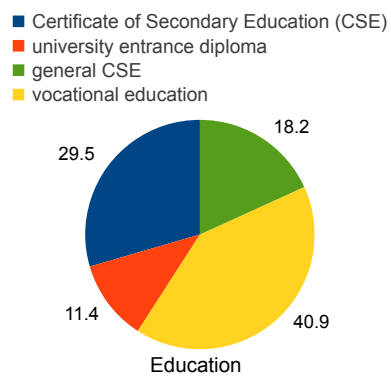

Figure 6. This figure lists the working units of the participants, the distribution over their native language and their education levels. 
Wrede et al., Kinesthetic Teaching of Redundant Robots in Task and Configuration Space

Table 1: Average ratings of general experience with the FlexIRob system.

\begin{tabular}{|c|c|c|c|}
\hline item German & item English & mean & SD \\
\hline \multicolumn{4}{|c|}{ General experience with the FlexIRob system } \\
\hline Einfachheit der Bedienung & Simplicity of handling & 2.07 & 0.82 \\
\hline Wie selbsterklärend fanden Sie die Bedienung? & How self-explanatory was the handling? & 2.18 & 0.95 \\
\hline Konnten Sie den Roboter aus dem Gefühl heraus bedienen? & Could you handle it instinctively? & 2.43 & 1.07 \\
\hline Bedienung körperlich anstrengend? & Was the handling physically exhausting? & 3.64 & 1.40 \\
\hline Mussten Sie bei der Bedienung des Roboters viel überlegen? & Did you have to think a lot during handling? & 3.66 & 1.18 \\
\hline Wie bedrohlich fanden Sie den Roboter? & Do you think the robot is threatening? & 4.27 & 1.06 \\
\hline Wie angenehm fanden Sie den Umgang mit dem Roboter? & Was the contact with the robot comfortable? & 2.13 & 0.97 \\
\hline Wie intelligent schätzen Sie den Roboter ein? & How intelligent do you rate the robot? & 1.78 & 0.80 \\
\hline Für wie zuverlässig halten Sie den Roboter? & How reliable do you think the robot is? & 2.07 & 0.90 \\
\hline Fanden Sie die Rückmeldungen hilfreich? & Do you think the feedback was helpful? & 1.57 & 0.95 \\
\hline \multicolumn{4}{|c|}{ Note: $1=$ yes $/$ very much, $5=$ no $/$ not at all } \\
\hline \multicolumn{4}{|c|}{ Robot assistance at work } \\
\hline Teile oder Werkzeuge reichen & Hand tools or parts & 1.50 & 0.63 \\
\hline Schwere Teile halten & Hold heavy parts & 1.37 & 0.65 \\
\hline Teile zusammenschrauben & Screw parts together & 1.93 & 0.73 \\
\hline Schwere Werkstücke wegräumen & Put heavy parts away & 1.36 & 0.64 \\
\hline
\end{tabular}

Table 2: Main effects.

\begin{tabular}{|l|llll|}
\hline & $\mathrm{F}$ & $\mathrm{df}$ & $\mathrm{p}$ & $\eta^{2}$ \\
\hline effect on simplicity of handling (without covariates) & 8.59 & 1.42 & $<.01$ & 0.17 \\
effect on reasonableness (without covariates) & 3.34 & 1.42 & $<.10$ & 0.07 \\
effect on simplicity of handling (with covariates) & 3.17 & 1.37 & $<.10$ & 0.08 \\
effect on instinctiveness of handling & 2.83 & 1.42 & $<.10$ & 0.06 \\
effect of knowing industrial robots on perceived threat & 7.58 & 1.42 & $<.01$ & 0.15 \\
\hline
\end{tabular}

A marginally significant effect could also be found for the operation mode. The non-assisted group $(\mathrm{M}=2.22, \mathrm{SD}=.85)$ rated it significantly less reasonable than did the assisted group $(\mathrm{M}=1.78$, $\mathrm{SD}=0.74)$. Again, low values indicate yes/very much, and high values indicate no/not at all.

The ANOVA was again computed with covariates that could have influenced the effects. Covariates were identified correlating all control variables with the dependent variables. Covariates included in the analysis were the length of time the participants had worked at HARTING, their experience with computers, their spatial vision, and their spatial imagination, as these variables significantly correlated with one or several of the dependent variables. Results showed a marginally significant effect for the simplicity of handling (see Figure 7). Again, participants in the non-assisted mode $(\mathrm{M}=2.37, \mathrm{SD}=1.12)$ rated the handling significantly more difficult than did participants in the assisted mode $(\mathrm{M}=1.88, \mathrm{SD}=0.71)$.

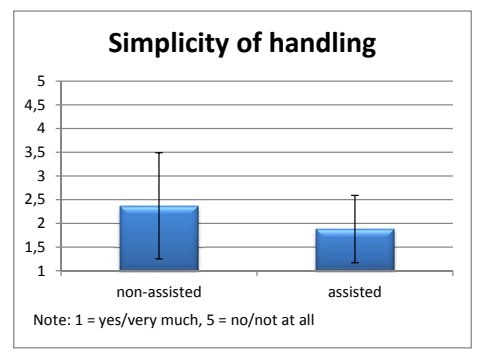

Another interesting result of this analysis is that the covari- during the programming. ate spatial vision was significantly related to the pleasantness of handling. Participants with better spatial vision rated the interaction more pleasant. For the values, see Table 3.

General Experience. As we were also interested in any differences concerning the general experience with our robot, an ANOVA was conducted with condition as the independent variable and all variables rating the general experience 
Table 3: Covariant effects.

\begin{tabular}{|c|c|c|c|c|c|}
\hline & F & $\mathrm{df}$ & $\mathrm{p}$ & $\eta^{2}$ & $\beta$ \\
\hline \multicolumn{6}{|l|}{ gender in the analysis } \\
\hline - of how self-explanatory the handling was & 4.33 & 1.34 & $<.05$ & 0.11 & 0.64 \\
\hline - of how physically exhausting the handling was & 4.78 & 1.34 & $<.05$ & 0.12 & 1.15 \\
\hline spatial vision in the analysis & & & & & \\
\hline - of pleasantness of handling & 6.52 & 1.37 & $<.05$ & 0.15 & 0.82 \\
\hline $\begin{array}{l}\text { - of how self-explanatory the handling was } \\
\text { prior }\end{array}$ & 4.54 & 1.34 & $<.05$ & 0.12 & 0.69 \\
\hline - of cognitive load & 4.89 & 1.34 & $<.05$ & 0.23 & 0.81 \\
\hline $\begin{array}{l}\text { - of how self-explanatory the handling was } \\
\text { number of known robots in the analysis }\end{array}$ & 6.79 & 1.34 & $<.05$ & 0.17 & -0.75 \\
\hline - of how threatening the robot was & 6.39 & 1.35 & $<.05$ & 0.16 & 0.16 \\
\hline
\end{tabular}

with the robot as dependent variables. Again, only means and standard deviations are described in the text; for other relevant values see Table 2. A marginally significant effect on the instinctiveness of handling could be found, indicating that the non-assisted group $(\mathrm{M}=2.71, \mathrm{SD}=1.01)$ rated the handling as less instinctive than did the assisted group $(\mathrm{M}=2.17, \mathrm{SD}=1.11)$. This difference is illustrated in Figure 8. This result is rather interesting, as participants were instructed to rate only the general experience with the robot and not the wire-loop game, where the experimental manipulation took place. This indicates that the positive effect was strong enough to influence the whole experience with the robot. Again, the ANOVA was computed a second time with covariates that could have influenced the effects. As before, the covariates were identified by correlating

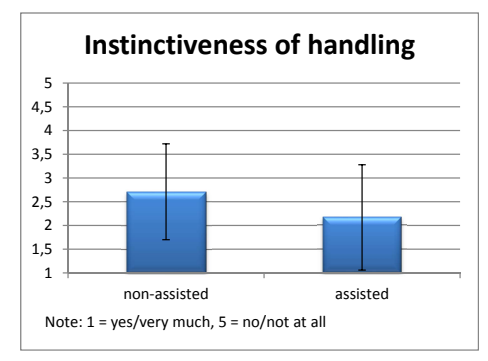

Figure 8. Average rating of instinctiveness of handling of the FlexIRob system in general.

all control variables with the dependent variables. Covariates included in the analysis were gender, computer experience, spatial vision, spatial imagination, prior experience with robots, number of robots known and the department at HARTING the participants worked at. This ANOVA did not reveal any significant differences regarding condition, which is in line with our expectations that there would be no significant differences concerning the general experience with the robot, as participants were instructed to rate the wire-loop game separately.

Nonetheless, interesting effects concerning the covariates could be found in the analysis of the general experience with the robot. For the sake of readability, the values are not integrated in the text; see Table 3 . The covariate gender was significantly related to how self-explanatory participants rated the handling: females rated the handling as less self-explanatory than males did. Even more interesting is the significant relation of gender to how physically exhausting the handling was rated, as males rated the handling more exhausting than females. The covariate spatial vision was again significant: Participants with better spatial vision rated the handling as more self-explanatory. The covariate prior experience with robots was also significantly related to how self-explanatory the handling was and how high the cognitive load during handling was. Interestingly, the cognitive load was higher for participants with more experience with robots, and they also rated the handling as less self-explanatory. Possible reasons for that need to be discussed. However, although these observations are interesting and hence worth reporting, a detailed discussion is not in the scope of this paper, and we therefore omit further elaboration of these effects here. 
5.1.4 Effect regarding the number of known robots The analysis of the general experience in the previous section also revealed that the number of known robots was significantly related to perceived threat. Participants who knew more robots rated the FlexIRob system used in the study as less threatening. We were interested in the reason for that and conducted another ANOVA, based on the assumption that knowledge of industrial robots had an effect on perceived threat. Whether participants were familiar with industrial robots or not was used as the independent variable, and the perceived threat was the dependent variable. A highly significant effect was found, indicating that participants familiar with industrial robots rated the robot significantly less threatening $(\mathrm{M}=4.62, \mathrm{SD}=.80)$ than did participants not familiar with industrial robots $(\mathrm{M}=3.78$,

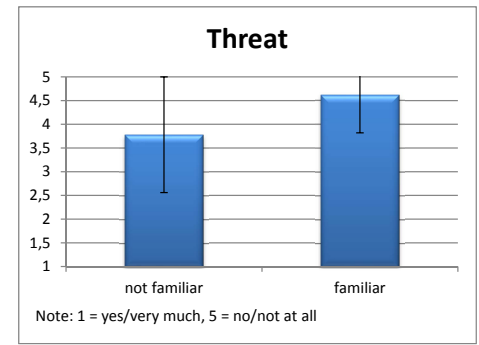

Figure 9. Average rating of perceived threat caused by the FlexIRob system in general.

$\mathrm{SD}=1.22$ ). This result is illustrated in Figure 9; for other values concerning this analysis see Table 2. Again, low values indicate high perceived threat, while high values indicate low perceived threat.

\subsection{Configuration Phase}

In the following, we present the results of the user study concerning the configuration task. The analysis in this (and the following) section is primarily based on the system data recorded during the experiments. To allow exact quantification of the collisions that occurred, we replayed, processed, and evaluated the recorded trajectories in a simulated environment, which was exactly modeled on the study setup. By this means, we could, for instance, exactly count the number of collisions with the environment without manual annotation of the recorded video data.

5.2.1 Illustration of different user behaviors We start this analysis with an illustration of the differences in the participants' user behaviors while interacting with the FlexIRob system. Indicated by the high standard deviations in Table 1, we hypothesize that these differences mostly consist of the cognitive load imposed on the participants during the task and the physical effort during the interaction. Regarding physical effort, these differences are well reflected in the recorded system data. As mentioned earlier, during the training phase the robot arm was controlled in joint impedance. For recording training data in a certain area of the workspace, the participants were encouraged to move the robot's end-effector in circular trajectories. Since the robot is commanded to stay in the user-defined posture, the participants had to exert force on it in order to perform these movements. These external forces, measured as estimated external torque in each joint, were recorded during the study and deliver an insight into the different user experiences. Whereas some participants did not hesitate to strongly push the robot from its commanded position and therefore induced torques up to $18 \mathrm{Nm}$ in some joints, other participants only slightly touched the end-effector and induced only about $2 \mathrm{Nm}$ as maximum joint torque. These different behaviors are also revealed by the task-space coordinates of the recorded training data. In Figure 10 (left), the recorded system data of three participants during the reconfiguration phase are shown. As illustrated in these figures, the cubic expansion of the recorded training data (solid green and red lines) varies from data with almost no variance in Figure 10(a) to data consisting of several circular movements with a diameter of up to $32 \mathrm{~cm}$, as in Figure 10(b).

Regarding the cognitive load, our hypothesis is that the inter-subject differences mainly relate to the problem of handling the redundant robot arm. Whereas some participants had no problem manipulating the KUKA LWR IV's joints in order to guide it to a certain end-effector position 
without colliding with obstacles in the environment, others seemed to think a lot and play around with the robot until they found a solution to the task (cf. Figure 10 (right) for some examples). These differences are also revealed by the participants' system data visualized in Figure 10 (left), where the red and green dashed lines show how the participants moved the robot's end-effector while trying to find a good training posture in the corresponding training area. In order to quantify these differences between the participants, we measured the time needed to guide the robot to a certain training area. For the first training area, these times varied from a minimum of ca. $8 \mathrm{~s}$ up to a maximum time of $104 \mathrm{~s}$, for the second training area, the minimum and maximum amount of time were 6 and $45 \mathrm{~s}$, respectively.

5.2.2 Defining metrics for evaluation According to (Steinfeld, Fong, \& Kaber, 2006), we evaluate the physical interaction of the participants with the FlexIRob system and their teaching success by accessing the system effectiveness and the system efficiency. The task effectiveness measures how well a predefined task is accomplished. The goal of the reconfiguration process is to teach the robot arm certain inverse kinematic solutions in different areas of its workspace in order to control it in the entire (predefined) workspace while avoiding the static environment. Thus, we identify two measures of task effectiveness: (a) accuracy of the performed test trajectory, which ideally consists of a straight trajectory between the training areas, and (b) the number of unintended contacts or collisions of the robot arm with its environment. Efficiency measures the time that is needed to complete a task. Here, we distinguish between the time that is spent on the kinesthetic teaching and the time needed for calculation of the estimated inverse kinematic model; that is, time that is solely consumed by the learning algorithm.

5.2.3 General configuration success As a result of the varying behavior of the participants described in Section 5.2.1, the training success also varied strongly. After each individual trial of a participant in the configuration phase (cf. Sec. 4.1.2), the FlexIRob system was commanded to move along a straight line in the work-space as a reference movement. Based on how well the trained system executed this reference trajectory, the results obtained after the third configuration trial with regard to effectiveness can be categorized as follows:

- 4 participants were unable to teach the system to produce no collisions and additionally only managed to achieve low accuracy of the test trajectory.

- 21 participants managed to train the system to produce no collisions but with low accuracy of the test trajectory. For an example, see Figure 10(a).

- 23 participants achieved high accuracy of the test trajectory but with collisions.

- 5 participants were able to teach the system such that high accuracy was achieved and no collisions occurred (see Figure 10(c)).

The impression that participants were able to either do the configuration accurately or collisionfree could also be supported by a correlation of $\mathrm{r}=-.58, \mathrm{p}<.01$. Thereby, the threshold of an appropriate task-space accuracy was set to $1 \mathrm{~cm}$.

The time needed for a single configuration procedure splits into time that is spend on the pHRI in the approaching and recording phases, and the computation time required by the learning algorithm. We report an average time of $54.1 \pm 24.8 \mathrm{~s}$ for the former and $5.3 \pm 1.0 \mathrm{~s}$ for the latter, which results in an average time requirement for a single entire configuration procedure of approximately $1 \mathrm{~min}$.

5.2.4 Users' performance development over trials In order to investigate the participants' performance development, we analyzed the error for the three configuration trials. Figure 11(a) shows the error development for each individual user over the three trials and corresponding box-plots indicating the distribution of errors at each trial. Interestingly, most of the users neither improved nor got worse from the first to the second trial, yielding a median Euclidean error of approximately 5 

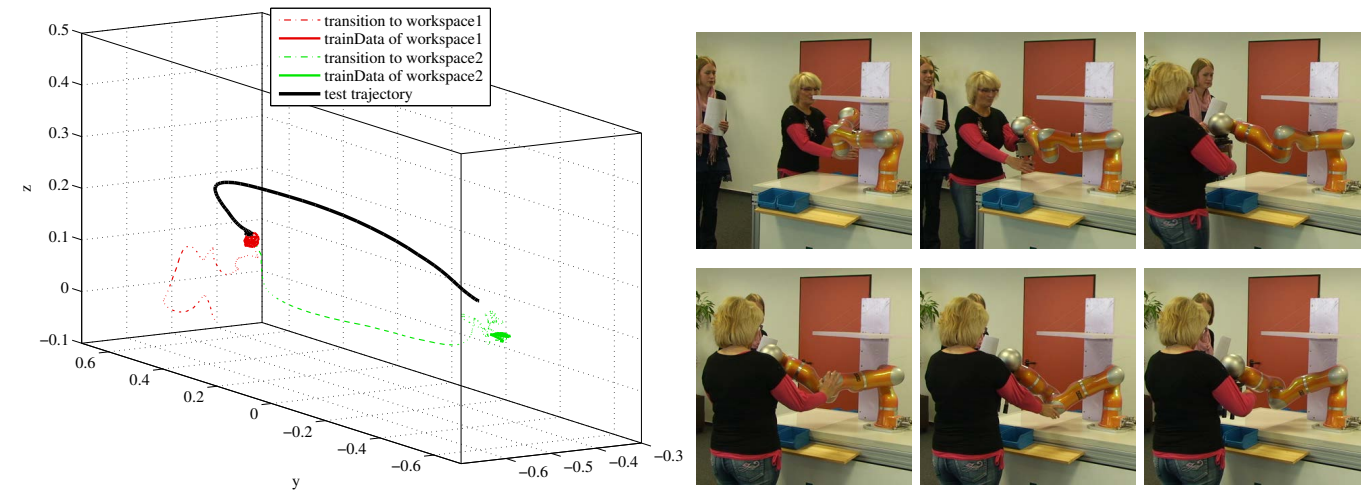

(a) Test person recording training data with only a low variance and guiding the robot arm through a singularity of the inverse kinematic. As a result, the accuracy of the performed test trajectory (should be a straight line in-between the two areas of the workspace) is poor.
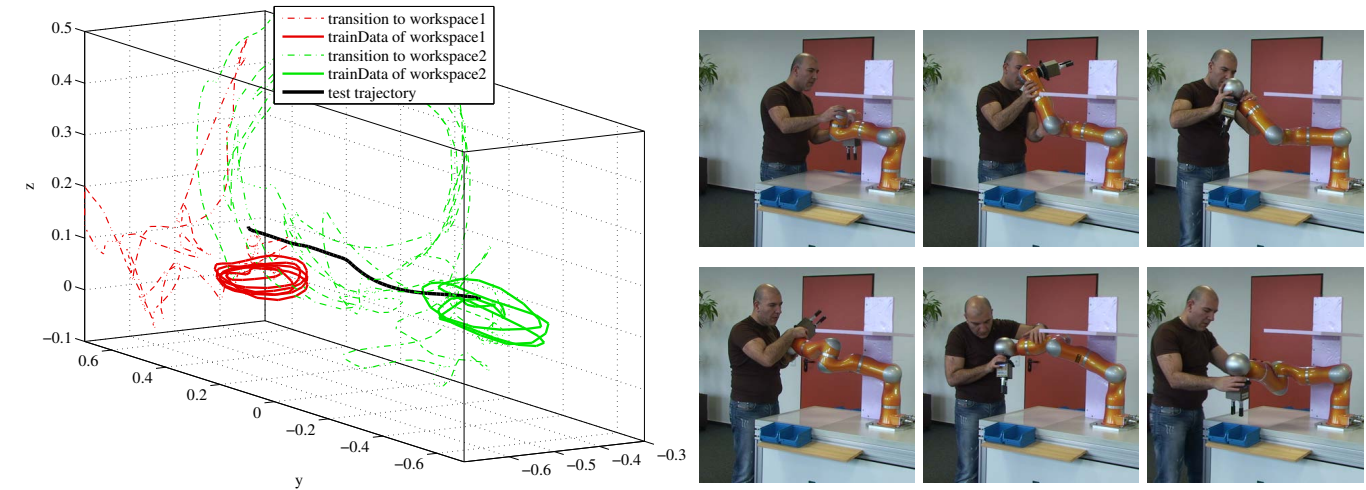

(b) Test person recording training data with a high variance but having problems guiding the robot arm from one workspace area to the other. Nevertheless, the performed test trajectory is more or less a straight line in between the two areas.
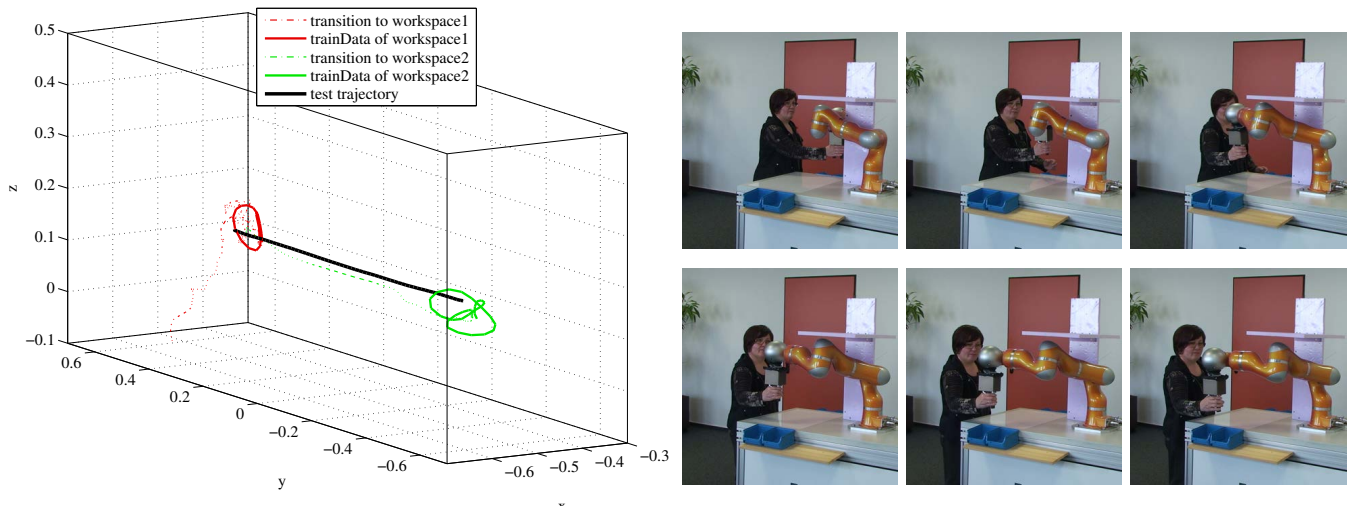

(c) Test person providing good training data with an appropriate variance and a valid combination of postures in both workspace areas. As a result, the robot is able to perform the test trajectory between the training areas with high accuracy.

Figure 10. Examples of different user behaviors during the configuration phase, varying in selected training postures, number of recorded training data, and training success. The 3-D data visualization on the left shows the recorded training data (solid green and red lines), the transition to the respective training area (dashed green and red lines), and the performed test trajectory of the robot (solid black line). On the right, camera snapshots give insight into the way the users guided the robot from the first training area to the second. 


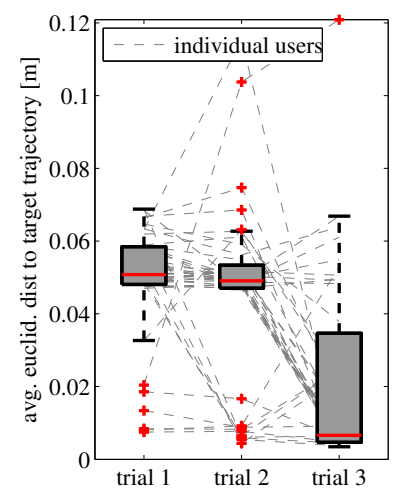

(a) Development of participants' task-space errors.

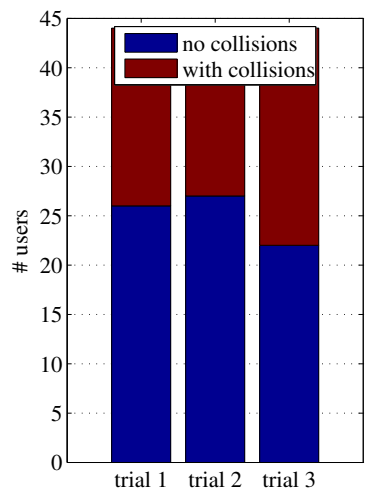

(b) Number of participants with collisions during testing.

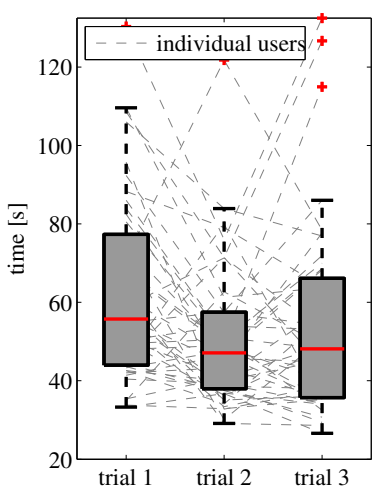

(c) Time needed by the participants for the configuration.

Figure 11. Development of the participants' performance over three trials of the configuration task.

$\mathrm{cm}$. However, in the third trial many users improved their performance significantly, such that the median user performance is below $1 \mathrm{~cm}$. Concerning the participants' ability to train the system to avoid the environmental obstacles, we could not measure any significant development over the three trials (see Figure 11(b)). As for the users' time needed for kinesthetic teaching, only a slight improvement was measurable from the first to the second trial (see Figure 11(c)).

\subsection{Programming Phase}

The results of Section 5.1.3 showed that participants that were assisted during the wire-loop game (group A) appraised the handling of the robot as easier than the non-assisted participants (group N) did. In addition, group A declared the settings of the robot to be more appropriate than group $\mathrm{N}$ did. In this section, we further analyze the programming phase on a system data level, where the differences regarding the two conditions are also well reflected.

Figure 12 shows the teach-in trajectories that were recorded by the participants during the wireloop game. It clearly reveals that the trajectories of group A (see Figure 12(a)) are smooth, very similar to each other, and close to the target, which was represented by the Styrofoam parcours during the study and is plotted in Figure 12 as black lines. In contrast, participants in group N (see Figure 12(b)) recorded trajectories that are jerky, deviate a lot from each other, and in some cases exhibit strong error in regard to the target trajectory.

In order to evaluate these differences, we again conducted an analysis of system effectiveness, which is assessed by task-space accuracy and avoidance of collisions with the environment, and system efficiency, which is measured by the time needed by the participants for the teach-in.

Since we did not explicitly encourage the participants to satisfy specific time constraints during the wire-loop game, we measured the geometrical similarity between the teach-in trajectories and the target. Geometrical shapes of trajectories are compared with the Procrustes analysis (Kendall, 1989). For this purpose, first the two trajectories are normalized in time; that is, we remove the velocity profile by re-sampling the data with equal velocity and equal number of points for both trajectories. Second, for comparison the trajectories are optimally superimposed by means of translation and rotation (we omit scaling in our analysis). In a final step, the goodness-of-fit criterion is calculated as the average Euclidean distance between the points of both trajectories. The results shown in Figure 13(a) clearly reveal the significantly higher task-space performance of participants 
in group A compared to those in group $\mathrm{N}$. The average Procrustes distance for group $\mathrm{N}$ measures $15.8 \pm 1.2 \mathrm{~cm}$, which means that even after optimal translation and rotation the geometrical shapes of teach-in trajectory and target trajectory on average differ by $15 \mathrm{~cm}$ per point. In contrast, the goodness-of-fit criterion for group A measures only $3.5 \pm 1.8 \mathrm{~cm}$. An ANOVA confirmed that the differences between the groups were significant (see Table 4). Concerning the number of collisions (see Figure 13(b)) with environmental obstacles, we report that for 22 of 24 participants in group $\mathrm{N}$ collisions occurred during the teach-in. In contrast, during the simulations we could detect only one of 24 participants in group A that induced unintended contacts between the robot arm and the environment.

The time needed by the participants for the teach-in is displayed in Figure 13(c). Again, the data reveal a significant advantage of group A. Whereas participants of group $\mathrm{N}$ needed $93.4 \pm 44.5 \mathrm{~s}$ on average, participants of group A required $44.9 \pm 13.9 \mathrm{~s}$; that is, only half the time for the same task.

In addition, we evaluated the trajectories' jerkiness systematically. An ANOVA showed that the recorded trajectories of the assisted users were significantly less jerky (given in $\mathrm{m} / \mathrm{s}^{3} ; \mathrm{M}=$ $0.00029, \mathrm{SD}=0.00005)$ than those of group $\mathrm{N}(\mathrm{M}=0.00039, \mathrm{SD}=0.00029)$; these results are given in Table 4. To assure the significant effects against influences from confounding variables, the ANOVAs were again executed with covariates. Covariates were identified correlating control variables from the questionnaire with the dependent variables. Spatial vision, spatial imagination, and the time participants had worked at HARTING were found to serve as possible covariates. As the new analysis included variables from the questionnaire, only the participants already included in the previous analyses of the questionnaire were included. The additional ANOVA, in which the influence of the covariates was controlled, showed several significant effects. It showed a significant effect for the average Procrustes distance: Group A showed significantly less distance (given in meters; $\mathrm{M}=0.03, \mathrm{SD}=0.02)$ than did group $\mathrm{N}(\mathrm{M}=0.16, \mathrm{SD}=0.12)$. Significant differences were also found for the time during the task: Group A was significantly faster (given in seconds; $\mathrm{M}=43.45, \mathrm{SD}=13.68)$ than group $\mathrm{N}(\mathrm{M}=95.24, \mathrm{SD}=46.27)$. Another significant effect was found for the jerkiness: the movements of participants in Group A were significantly less jerky (given in $\left.\mathrm{m} / \mathrm{s}^{3} ; \mathrm{M}=0.00022, \mathrm{SD}=0.00005\right)$ than those of participants in group $\mathrm{N}(\mathrm{M}=0.00041$, $\mathrm{SD}=0.00030$ ). For more information see Table 4.

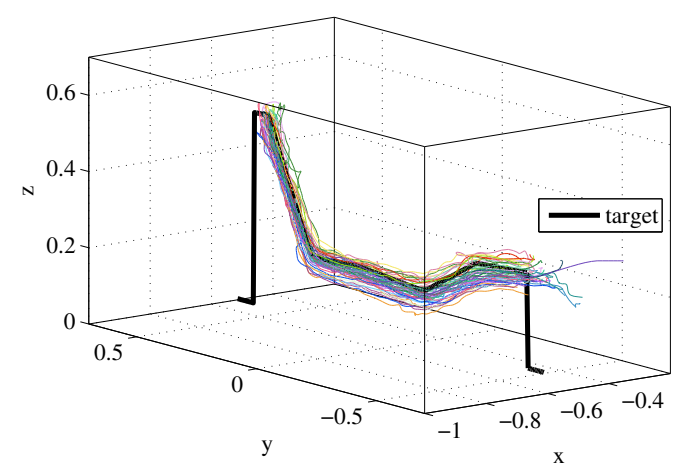

(a) Group A (assisted)

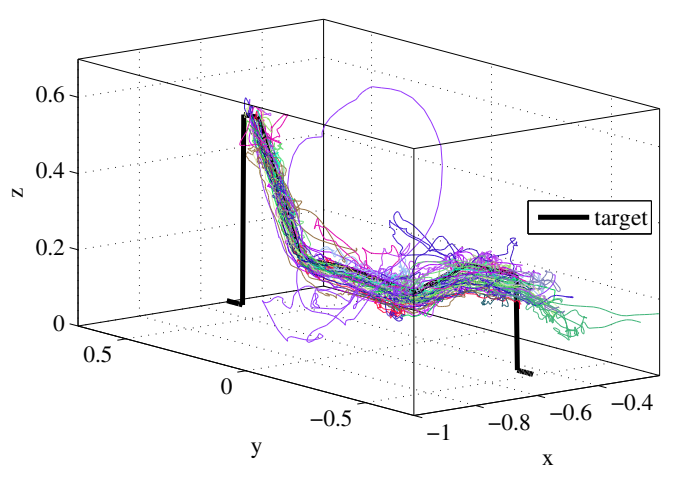

(b) Group N (non-assisted)

Figure 12. Teach-in trajectories of the participants during the wire-loop game. 


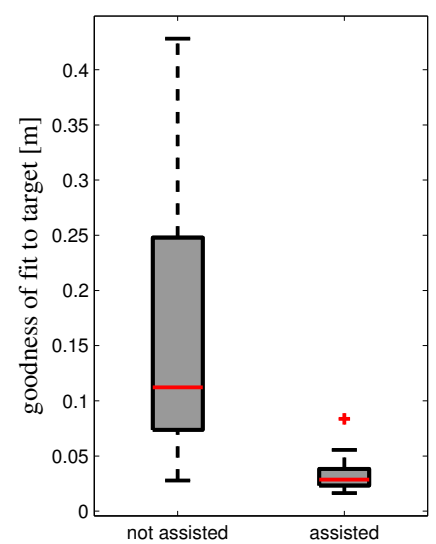

(a) Results of the Procrustes analysis comparing the shape of the participants' teach-in trajectories to the target trajectory.

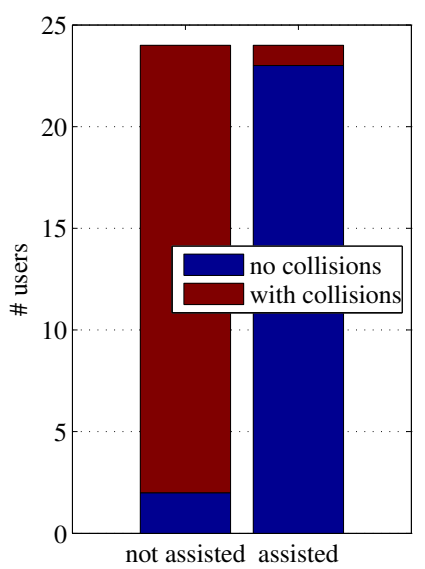

(b) Number of participants with and without collisions.

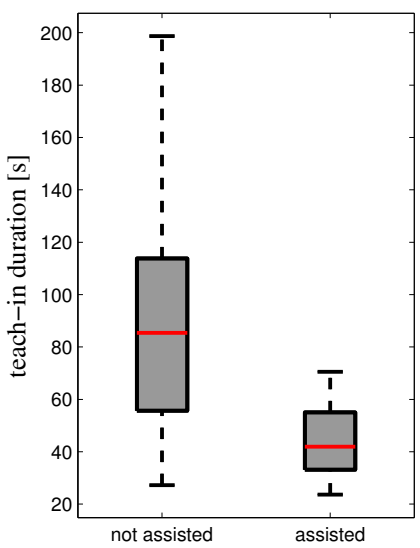

(c) Time needed by the participants for the teach-in.

Figure 13. Comparison of the participants' performance during the teach-in of the wire-loop game.

Table 4: Main effects of the condition on the system data.

\begin{tabular}{|l|llll|}
\hline & $\mathrm{F}$ & $\mathrm{df}$ & $\mathrm{p}$ & $\eta^{2}$ \\
\hline average Procrustes distance (without covariates) & 24.82 & 1,46 & $<.0001$ & 0.35 \\
time wire-loop (without covariates) & 25.91 & 1,46 & $<.0001$ & 0.36 \\
max. jerkiness (without covariates) & 7.97 & 1,46 & $<.001$ & 0.15 \\
average Procrustes distance (with covariates) & 15.57 & 1,37 & $<.0001$ & 0.30 \\
time wire-loop (with covariates) & 21.35 & 1,37 & $<.0001$ & 0.37 \\
max. jerkiness (with covariates) & 4.71 & 1,37 & $<.05$ & 0.11 \\
\hline
\end{tabular}

\section{Discussion}

Based on the quantitative analysis of the systems study presented in the preceding section, it is now possible to set these results in relation to our expectations formulated in Section 4.

\subsection{General Experience}

Regarding the general experience with our FlexIRob system, we expected the physical interaction to be easy and comfortable, not threatening to the users, and self-explanatory in particular for people with good spatial vision abilities. In general, these expectations were confirmed by our results. The results also confirmed that the pleasantness of handling was indeed significantly related to good spatial vision. This underlines the importance of immediate feedback in order to mitigate this effect for people with less sophisticated spatial vision.

In contrast to these confirmations of our predictions, we are - positively - surprised that the subjects rated the robot as non-threatening despite the workers' extensive exposure to safety instructions about industrial machinery. Along these lines, workers who had practical experience with industrial robots felt FlexIRob was significantly less threatening. We assume that both effects can be explained through the smooth and spontaneous force feedback of this robot in direct physical interaction. Participants with prior experience in industrial robotics rated the interaction less selfexplanatory and reported a higher cognitive load during the interaction as the analysis of covariates 
Wrede et al., Kinesthetic Teaching of Redundant Robots in Task and Configuration Space

indicates. The reason for this is unclear, but we hypothesize that this effect is due to mismatched expectations regarding the human-robot interaction. Further analysis will be required on this point.

\subsection{Interactive Constraint Configuration}

Considering the feasibility of task-independent configuration for inexperienced users, our expectations in terms of time required for reconfiguration, collision avoidance in joint space, and the taskspace accuracy of the learned hierarchical controller were not entirely confirmed. Nevertheless, the objective results show that the configuration of the redundancy resolution through kinesthetic teaching and the learning of the inverse kinematics mapping can be done in less than $2 \mathrm{~min}$, and that at least half of the users could perform the kinesthetic teaching accurately such that collisions with the environment were avoided. Hence, we think that kinesthetic teaching is feasible and useful also beyond the typical trajectory level.

Further analysis on the desired characteristics of the recorded training data is required. While the accuracy of the resulting hierarchical controller is in some user trials very good, some outliers degrade the overall performance statistics. Besides this, no significant progress in task fulfillment could be measured between the three user trials. Our interpretation of these results is that future work on kinesthetic teaching for learning robots needs to provide improved spontaneous feedback; for example, on the quality of the gathered training data and to utilize further intrinsic properties of the robot system for a more efficient tutoring interaction.

\subsection{Assisted Trajectory Programming}

Decomposing the interaction required to fully reconfigure a redundant robot into configuration and programming phases allows the use of the learned hierarchical controller with redundancy resolution in the proposed assisted gravity compensation mode. We expected a better interaction experience and improved teach-in performance using this new interaction mode for kinesthetic teaching of task-space trajectories. These expectations were completely fulfilled. With regard to the interaction experience, we found a highly significant effect in the subjective assessment for ease of handling and a marginal effect for instinctiveness of the system in favor of the assisted mode. Furthermore, the non-assisted group rated the robot's behavior significantly less reasonable than the assisted group. Beyond that, the results originating from the questionnaire are supported through objective system measurements that significantly confirm our expectations; for example, about accuracy in terms of Cartesian error and jerkiness. Collisions with the environment were almost completely eliminated. The single remaining collision can be attributed to a robot failure but is not related to the interaction concept used in this mode. Last but not least, participants in the assisted group managed to kinesthetically teach the reference trajectory in only half the time required by the non-assisted group. These results underline that the decomposition and assistance features realized in this concept provide a measurable benefit for the kinesthetic teaching of task-space trajectories with redundant robots.

\section{Conclusion}

In this article, we presented an integrated interaction and machine learning approach for reconfiguration of redundant manipulators. In contrast to existing approaches, the reconfiguration interaction is split into a task-independent configuration phase, where invariant constraints can be implicitly taught, and a programming phase, where task-space trajectories are specified. Reconfiguration of the system can thus be achieved by users without in-depth robotics knowledge, using kinesthetic teaching to gather training data implicitly containing constraints present in the environment or required by the intended task. The learning process uses a data-driven and model-free approach for training a neural network, which becomes an embedded part of a hierarchical controller effectively used in an assisted gravity compensation mode to support the user in the programming interaction. 
Within this interaction mode, the system controls the joints of the robot and lets the user fully concentrate on the task at hand by guiding the end-effector of the robot.

To evaluate this decomposition and our implementation of it in an integrated system based on the KUKA LWR IV robot, we conducted a systems study with 49 participants from the HARTING technology group, a medium-sized manufacturing company. Within this study, not only subjective data from questionnaires but also objective data gathered directly from the live system was analyzed for significant effects regarding the test condition (assisted/non-assisted teach-in). To the best of our knowledge, no such study on the reconfiguration of a redundant robot platform that is commercially available and uses kinesthetic teaching exists in the literature. We found significant effects in the study confirming that the decomposition into configuration and programming phases, as well as the introduced assisted gravity compensation mode, do have a positive impact on the usability and performance of programming trajectories on redundant robots. Furthermore, this work demonstrates that the transfer of implicit knowledge of the human tutor through kinesthetic teaching (e.g., about environmental constraints) is possible and is useful even beyond the trajectory level. In this context, it provides a much more efficient way to encode the environment in joint-space compared to the explicit constraint modeling in other approaches.

Beyond that, the objective results show that the new assistance mode allows human tutors to teach trajectories in the task-space faster, with higher accuracy and less cognitive load compared to the standard approach used by the control group. Last but not least, this decomposition of the required reconfiguration allows for generalization to robots with higher degrees of freedom even if a kinesthetic teaching of the constraints is not possible. Still, the assisted gravity compensation concept could be used to easily program tasks with redundant robots. Future work will consider further optimization of the learning approach used in the configuration phase and improved feedback in the different interaction modes.

\section{Acknowledgments}

This work was partly funded by the ECHORD experiment "Model-free flexible trajectory generation" $(\mathrm{MoFTaG})^{6}$ and by the European Community's Seventh Framework Programme FP7/20072013 Challenge 2 Cognitive Systems, Interaction, Robotics under grant agreement No 248311 AMARSi. We are grateful to HARTING ${ }^{7}$ for allowing us to place the FlexIRob system at their production site and for facilitating 50 workers to participate in the study.

\section{Appendix: Stiffness and Damping Values}

Table 5: Stiffness and damping values chosen for the different interaction control modes.

\begin{tabular}{lccc} 
Mode & stiffness & damping & inertia \\
\hline controlled gravity compensation & $20.0 \frac{N m}{r a d}$ & $0.7 \frac{N m * s}{r a d}$ & 0.1 \\
hierarchical control & $300.0 \frac{N m}{r a d}$ & $0.95 \frac{N m * s}{r a d}$ & - \\
compliant recording & $80.0 \frac{N m}{r a d}$ & $0.7 \frac{N m * s}{r a d}$ & - \\
compliant execution & $300.0 \frac{N m}{r a d}$ & $0.95 \frac{N m * s}{r a d}$ & - \\
assisted gravity compensation & $50.0 \frac{N m}{r a d}$ & $0.7 \frac{N m * s}{r a d}$ & 0.5
\end{tabular}

\footnotetext{
${ }^{6}$ http://www.echord.info/wikis/website/moftag

${ }^{7}$ HARTING technology group - http://www.HARTING.com. For further information please contact gisela.eickhoff@HARTING.com
} 
Wrede et al., Kinesthetic Teaching of Redundant Robots in Task and Configuration Space

\section{References}

Akgun, B., Cakmak, M., Yoo, J., \& Thomaz, A. (2012). Trajectories and keyframes for kinesthetic teaching: A human-robot interaction perspective. In Proceedings of the seventh annual ACM/IEEE international conference on Human-Robot Interaction (HRI2012), 391-398. http://dx.doi.org/10.1145/2157689.2157815.

Alami, R., Albu-Schaeffer, A., Bicchi, A., Bischoff, R., Chatila, R., \& ... Hirzinger, G. (2006). Safe and dependable pHRI in anthropic domains: State of the art and challenges. In Proceedings of the 4th IARP/IEEE-RAS/EURON Workshop on Technical Challenges for Dependable Robots in Human Environments.

Albu-Schäffer, A., Haddadin, S., Ott, C., Stemmer, A., Wimböck, T., \& Hirzinger, G. (2007). The DLR lightweight robot: Design and control concepts for robots in human environments. Industrial Robot, 34(5), 376-385, http://dx.doi.org/10.1108/01439910710774386.

Albu-Schäffer, A., Ott, C., \& Hirzinger, G. (2007). A Unified Passivity Based Control Framework for Position, Torque and Impedance Control of Flexible Joint Robots. In S. Thrun, R. Brooks, \& H. DurrantWhyte (Eds.), Robotics research (Vol. 28, pp. 5-21). Berlin, Heidelberg: Springer Berlin Heidelberg, http://dx.doi.org/10.1007/978-3-540-48113-3_2.

Artemiadis, P. K., Katsiaris, P. T., \& Kyriakopoulos, K. J. (2010). A biomimetic approach to inverse kinematics for a redundant robot arm. Autonomous Robots, 29(3-4), 293-308, http://dx.doi.org/10.1007/s10514010-9196-x.

Billard, A., Calinon, S., Dillmann, R., \& Schaal, S. (2007). Chapter 59: Robot Programming by Demonstration. In B. Siciliano \& O. Khatib (Eds.), Springer Handbook of Robotics (pp. 1371-1394). Springer Berlin Heidelberg, http://dx.doi.org/10.1007/978-3-540-30301-5_60.

Bischoff, R., Kurth, J., Schreiber, G., Koeppe, R., Albu-Schaeffer, A., Beyer, A., et al. (2010). The KUKADLR Lightweight Robot arm - a new reference platform for robotics research and manufacturing. Joint 41 st International Symposium on Robotics and 6th German Conference on Robotics, 741-748.

Breazeal, C., Siegel, M., Berlin, M., Gray, J., Grupen, R., Deegan, P., et al. (2008). Mobile, dexterous, social robots for mobile manipulation and human-robot interaction. In Special Interest Group on Graphics and Interactive Techniques. http://dx.doi.org/10.1145/1401615.1401642.

Calinon, S., \& Billard, A. (2007a). Incremental learning of gestures by imitation in a humanoid robot. In Proceedings of the ACM/IEEE international conference on Human-robot interaction (pp. 255-262). New York, NY, USA, http://dx.doi.org/10.1145/1228716.1228751: ACM.

Calinon, S., \& Billard, A. (2007b). What is the teacher's role in robot programming by demonstration? Toward benchmarks for improved learning. Interaction Studies, 8(3), 441-464.

Conkur, E., \& Buckingham, R. (1997). Clarifying the definition of redundancy as used in robotics. Robotica, 15(5), 583-586, http://dx.doi.org/10.1017/S0263574797000672.

Daimler AG. (2009). Leichtbauroboter im Piloteinsatz im Mercedes-Benz Werk Untertürkheim (Lightweight robots employed in pilot application at Mercedes-Benz site Untertürkheim), Press release. Daimler AG, Available at: http://goo.gl/RSeYl.

De Santis, A., Siciliano, B., De Luca, A., \& Bicchi, A. (2008, March). An atlas of physical human-robot interaction. Mechanism and Machine Theory, 43(3), 253-270, http://dx.doi.org/10.1016/j.mechmachtheory.2007.03.003.

European Strategic Robotics Platform. (2009). Robotic visions to 2020 and beyond - The strategic research agenda (SRA) for robotics in Europe (Tech. Rep.). European Robotics Technology Platform, Brussels. Available online at: http://www.robotics-platform.eu/cms/upload/SRA/2010-06_SRA_A4_low.pdf.

Gribovskaya, E., Kheddar, A., \& Billard, A. (2011). Motion learning and adaptive impedance for robot control during physical interaction with humans. In 2011 IEEE International Conference on Robotics and Automation (ICRA) (pp. 4326 - 4332). http://dx.doi.org/10.1109/ICRA.2011.5980070.

Grupen, R., \& Huber, M. (2005). A framework for the development of robot behavior. In AAAI spring symposium series: Developmental robotics. Stanford University. (Available at: http://citeseerx.ist.psu.edu/viewdoc/summary?doi=10.1.1.177.5527)

Grzesiak, A., Becker, R., \& Verl, A. (2011). The bionic handling assistant: A success story of additive manufacturing. Assembly Automation, 31(4), 329 - 333. 
Wrede et al., Kinesthetic Teaching of Redundant Robots in Task and Configuration Space

Infante, M., \& Kyrki, V. (2011). Usability of force-based controllers in physical human-robot interaction. In HRI '11: Proceedings of the 6th International Conference on Human-Robot Interaction (pp. 355-362). http://dx.doi.org/10.1145/1957656.1957790.

Ito, M., \& Tani, J. (2004). On-line imitative interaction with a humanoid robot using a dynamic neural network model of a mirror system. Adaptive Behavior, 12(2), 93-115, http://dx.doi.org/10.1177/105971230401200202.

Jordan, M. I. (1992). Constrained supervised learning. Journal of Mathematical Psychology, 36(3), 396-425, http://dx.doi.org/10.1016/0022-2496(92)90029-7.

Kendall, D. G. (1989). A survey of the statistical of shape theory. Statistical Science, 4(2), 87-99, http://dx.doi.org/10.1214/ss/1177012582.

Kulic, D., Ott, C., Lee, D., Ishikawa, J., \& Nakamura, Y. (2011). Incremental learning of full body motion primitives and their sequencing through human motion observation. The International Journal of Robotics Research, 31(3), 330-345, http://dx.doi.org/10.1177/0278364911426178.

Lalo, W., Brandt, T., Schramm, D., \& Hiller, M. (2008). A linear optimization approach to inverse kinematics of redundant robots with respect to manipulability. In The 25th International Symposium on Automation and Robotics in Construction. ISARC-2008 (pp. 175-180). Vilnius, Lithuania: Vilnius Gediminas Technical University Publishing House Technika, http://dx/doi.org/10.3846/isarc.20080626.175.

Lee, D., \& Ott, C. (2010). Incremental motion primitive learning by physical coaching using impedance control. In IEEE/RSJ International Conference on Intelligent Robots and Systems (IROS) (pp. 4133 4140). Taipeh, Taiwan, http://dx.doi.org/10.1109/IROS.2010.5650519.

Levas, A., \& Selfridge, M. (1984). A user-friendly high-level robot teaching system. In IEEE International Conference on Robotics and Automation (Vol. 1, pp. 413-416). http://dx.doi.org/10.1109/ROBOT.1984.1087193.

Muehlig, M., Steil, J. J., \& Gienger, M. (2012). Interactive imitation learning of object movement skills. Autonomous Robots, 32(2), 97-114, http://dx.doi.org/10.1007/s10514-011-9261-0.

Neumann, K., Rolf, M., \& Steil, J. (2010). Learning inverse kinematics for pose-constraint bi-manual movements. In Proceedings of the 11th international conference on Simulation of adaptive behavior: From animals to animats, 478-488, http://dx.doi.org/10.1007/978-3-642-15193-4_45.

Nordmann, A., Emmerich, C., Rüther, S., Lemme, A., Wrede, S., \& Steil, J. (2012). Teaching nullspace constraints in physical human-robot interaction using reservoir computing. In IEEE International Conference on Robotics and Automation (ICRA) (pp. 1868-1875). http://dx.doi.org/10.1109/ICRA.2012.6225170.

Nordmann, A., Rolf, M., \& Wrede, S. (2012). Software abstractions for simulation and control of a continuum robot. In SIMPAR 2012 - Simulation, Modeling and Programming for Autonomous Robots. Lecture Notes in Computer Sience (Vol. 7628, pp. 113-124). Springer. Tsukuba, Japan. http://dx.doi.org/10.1007/9783-642-34327-8_13.

Sauser, E., Argall, B., Metta, G., \& Billard, A. (2012). Iterative learning of grasp adaptation through human corrections. Robotics and Autonomous Systems, 60(1), 55-71, http://dx.doi.org/10.1016/j.robot.2011.08.012.

Schaal, S., Ijspeert, A., \& Billard, A. (2003). Computational approaches to motor learning by imitation. Philosophical Transactions of the Royal Society of London Series B Biological sciences, 358(1431), 537-547, http://dx.doi.org/10.1098/rstb.2002.1258.

Schreiber, G., Stemmer, A., \& Bischoff, R. (2010). The fast research interface for the KUKA Lightweight Robot. IEEE ICRA 2010 Workshop on Innovative Robot Control Architectures, 15-21.

Siciliano, B. (1990). Kinematic control of redundant robot manipulators: A tutorial. Journal of Intelligent and Robotic Systems, 3(3), 201-212, http://dx.doi.org/10.1007/BF00126069.

Steinfeld, A., Fong, T., \& Kaber, D. (2006). Common metrics for human-robot interaction. In HRI '06: Proceedings of the 1st ACM SIGCHI/SIGART Conference on Human-Robot Interaction (pp. 33-40). http://dx.doi.org/10.1145/1121241.1121249.

Tsagarakis, N. G., Sardellitti, I., \& Caldwell, D. G. (2011). A new variable stiffness actuator (CompAct-VSA): Design and modelling. In 2011 IEEE/RSJ International Conference on Intelligent Robots and Systems (IROS) (pp. 378-383). http://dx.doi.org/10.1109/IROS.2011.6048698. 
Vijayakumar, S., D’souza, A., Shibata, T., Conradt, J., \& Schaal, S. (2002). Statistical learning for humanoid robots. Autonomous Robots, 12, 55-69, http://dx.doi.org/10.1023/A:1013258808932.

Wienke, J., Klotz, D., \& Wrede, S. (2012). A Framework for the acquisition of multimodal human-robot interaction data sets with a whole-system perspective. In Proceedings of the LREC Workshop on Multimodal Corpora for Machine Learning (pp. 46-49). Istanbul, Turkey. (Available at: http://bit.ly/VKT9eY)

\footnotetext{
Authors' names and contact information: Sebastian Wrede, CoR-Lab, Bielefeld University, Germany. Email: swrede@cor-lab.uni-bielefeld.de. Christian Emmerich, CoR-Lab, Bielefeld University, Germany. Email: cemmeric@ cor-lab.uni-bielefeld.de. Ricarda Grnberg, CoR-Lab, Bielefeld University, Germany. Email: ricarda.gruenberg@googlemail.com. Arne Nordmann, CoR-Lab, Bielefeld University, Germany. Email: anordman@cor-lab.uni-bielefeld.de. Agnes Swadzba, CoRLab, Bielefeld University, German. Email: aswadzba@cor-lab.uni-bielefeld.de. Jochen Steil, CoRLab, Bielefeld University, German. Email: jsteil@cor-lab.uni-bielefeld.de.
} 